\title{
La sociología del trabajo en la Universidad de la Habana
}

EUCLIDES CATÁ GUILARTE*

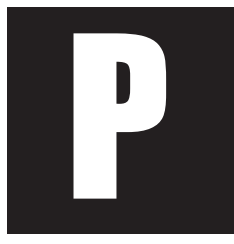

ara comprender el desarrollo de la Sociología del trabajo en la Universidad de La Habana (UH) como teoría especial, es necesario tener en cuenta las coyunturas históricas por las que han atravesado las ciencias sociales en el país, y especialmente la sociología.

La sociología como carrera independiente surge en el país en 1959, en la Universidad de Oriente. Esta fecha coincide con el surgimiento de la carrera en otros países latinoamericanos. En condiciones políticas sociales diversas se fundan las escuelas y facultades de Sociología en América Latina (Colombia, 1959; Chile, 1958; Buenos Aires, 1957). Aparecen los sociólogos y sus congresos nacionales. Se realizan publicaciones sociológicas y se avanza en la institucionalización de la sociología (Cecilioa Bobes, 1990; Cataño, 1986 e 1996/1997).

En Cuba, el proceso de institucionalización de la sociología ha sido irregular. La Escuela de Sociología de la Universidad de Oriente desaparece con la Reforma Universitaria de 1962. Su última graduación fue en 1966 y el número de egresados fueron solamente 34. En 1968 se crea el Departamento de Sociología en la Facultad de Humanidades de la Universidad de

* Profesor de Sociología. Departamento de Sociología. Universidad de La Habana. San Lázaro y L, Vedado. La Habana. 
La Habana, el que se responsabiliza a partir de 1969 de la formación de los sociólogos en el país. En el año 1976, con la creación del Ministerio de Educación Superior y los cambios institucionales en la UH, desaparece el Departamento de Sociología y sus estudiantes pasan en plan de liquidación, a la Facultad de Filosofía e historia de nueva creación. Esta última mantiene "oficialmente" la especialización de sociología en sus planes de estudios, pero no es hasta 1986-1987 que se hace realidad con el primer grupo de estudiantes de filosofía.

Como parte del proceso de rectificación de errores, la dirección del país planteó la necesidad de reevaluar la concepción de la formación de profesionales en la educación superior, pues ésta se había orientado a la búsqueda de un egresado de $\mathrm{n}$ grado de especialización tal que dificultaba el aprovechamiento y ubicación del mismo. Se orientó la confección de un Plan de Estudios que lograra un egresado de perfil amplio para que la especialización la realizara en la producción y en función de la esfera en que trabajara. De las carreras aprobadas, reaparece la sociología. La que se reinicia como carrera en el curso 1990-1991. ${ }^{1}$

El resumen que aquí presento acerca de la formación del sociólogo en el país indica la falta de coherencia en la política seguida en su formación lo que ha incidido en el proceso de su institucionalización. ${ }^{2}$

Las circunstancias que hacen que la sociología se haya desarrollado de forma intermitente en el país han sido explicadas en ponencias, artículos y otros estudios realizados. ${ }^{3}$ A grandes rasgos se concluye que:

\footnotetext{
1 Un análisis más detallado acerca de los estudios de sociología se encuentra en la ponencia: «La experiencia cubana en la formación del sociólogo», presentada por Euclides Catá y Mariana Ravenet en el XVIII Congreso de ALAS, Ciudad de la Habana, 1991.

2 La institucionalización no sólo tiene que ver con la formación de pregrado y posgrado en la Educación Superior sino también con eventos propios, participación de sociólogos en comisiones de expertos para el trabajo de políticas en organismos gubernamentales, intervención en eventos internacionales y relaciones con la comunidad científica internacional, existencia de asociaciones profesionales nacionales y de instituciones especializadas en la realización de investigaciones sociológicas y/o de servicios de sociología aplicada. C f. Mayra Espino Prieto, «Tropiezos y oportunidades de la sociología cubana», Temas, núm. 1, 1995.

3 De los trabajos dedicados al estudio de la sociología posterior al triunfo de la Revolución están: Helen Ochoa Calvo, «Consideraciones generales sobre la influencia de la formación, en las perspectivas de utilización de los sociólogos», tesis de
} 
1. La desaparición de la primera escuela se debe a la preeminencia que se les da a las ciencias duras en detrimento de las ciencias sociales.

2. La apertura de la carrera en la UH en 1969 como consecuencia del desarrollo que iban alcanzando las investigaciones sociales multidisciplinarias en el ámbito de un omento de reflexión y desarrollo de un pensamiento marxista creador en el país.

3. La desaparición de la carrera (curso 1976-1977) debido a la elección de elementos sustanciales del modelo soviético en diferentes esferas de la vida social cubana y a la generalización de la enseñanza del marxismo que admitía sólo la filosofía, la economía política y el comunismo científico sin que aquí encontraran cabida ni la sociología ni otras ciencias sociales.

4. La reapertura como especialización en filosofía (curso 1986-1987) y más tarde como carrera (1990-1661), debido al proceso de rectificación de la segunda mitad de los ochenta y a la apertura que marcó el Ilamamiento al IV Congreso del Partido con relación a las ciencias sociales y el papel que le asignan los documentos del IV Congreso a las Ciencias Sociales y Humanísticas. ${ }^{4}$

La forma discontinua en que se ha desarrollado la docencia de la sociología como carrera en el país ha influido de forma adversa en la formación de profesionales en esta ciencia, en la sistematicidad y alcance de sus programas de estudio, en la estabilidad y desarrollo científico y docente de los profesores, en las relaciones académicas con otras instituciones en el exte-

\footnotetext{
licenciatura, 1994: Aymara Hernández Morales, «Sociología de la Sociología: un análisis crítico de esta ciencia en Cuba a partir de 1959», tesis de licenciatura, 1995: Euclides Catá y Mariana Prieto, art. Cit. Otros trabajos que explícita o implícitamente incluyen el devenir de la sociología son: José A. Toledo y Jorge Núçez Jover, «Las ciencias sociales en el proceso de construcción del socialismo en Cuba. Introducción a su análisis», Cuba Socialista, Núm. 3, 1990: Aurelio Alonso Tejada, «Marxismo y espacio de debate en la Revolución Cubana», Temas, núm. 3, 1995,

4 «Nuestras ciencias sociales y humanísticas, debilitadas en el pasado por la falta de auténtico debate científico y la tendencia a copiar y repetir supuestas verdades establecidas por otros, están llamadas a resurgir y hacer sentir su papel en la investigación, el conocimiento y la transformación de nuestras realidades sociales». IV Congreso del Partido, «iEl futuro de nuestra patria será un eterno Baraguá!», Granma, 18 de marzo de 1990, p. 5.
} 
rior y en la producción y actualización de la literatura en el campo de las ciencias sociales y especialmente de la sociología.

\section{Docencia de la Sociología del Trabajo en la Universidad de la Habana}

La docencia de la Sociología del Trabajo ha estado presente en todos los planes de estudio. Incluyendo el de la primera escuela de la Universidad de Oriente. En la UH inicia la impartición de esta asignatura el profesor Ricardo J. Machado Beremúdez. ${ }^{5}$

En la elaboración de los planes de estudio en esta área ha influido, al igual que en las demás teorías sociológicas especiales, el desarrollo y el enfoque de las ciencias sociales en el país, las relaciones de la institución y sus docentes, tanto en el ámbito nacional como internacional y el nivel de información y área de influencia.

Los programas elaborados de Sociología del Trabajo en los setenta incluían temáticas referidas a su objeto de estudio y categorías. La sociedad contemporánea y la Revolución Científico-Técnica; los factores subjetivos en la producción; sistema de dirección y política de cuadros, y asuntos que tienen que ver con la conducta de los trabajadores como la disciplina laboral. En todas las materias posibles se reflexionaba acerca de la sociedad cubana.

Si bien es cierto que se logró un buen nivel en el desarrollo de estos programas, la bibliografía disponible ${ }^{6}$ las experiencias analizadas provenían de los ex países socialistas. Ello se debe a la política que imperaba en esos momentos acerca de las ciencias sociales, la desaparición de la escuela de

\footnotetext{
5 Ricardo Machado Bermúdez, sociólogo, obtuvo el grado de Dr. En Ciencias Económicas en la Universidad de Humboldt de Berlín (ex RDA). La continuó en la docencia Enrique Iñigo Bajos. Desde el curso 1988-1989 imparto también esta asignatura. 6 Algunas de las obras utilizadas: Colectivo de Autores, Industria y Trabajo en la URSS, La Habana, Ciencias Sociales, 1975; Colectivo de Autores (alemanes), El dirigente, el colectivo, la personalidad, C. de La Habana, Ciencias Sociales, 1991, y Richard
} 
sociología y con ello la ruptura de los lazos establecidos con algunas instituciones latinoamericanas.

Es necesario aclarar que la literatura mayormente utilizada proveniente principalmente de la ex URSS y ex RDA, en muchos casos incluían información y contenidos teórico-metodológicos e instrumentales de valor para la docencia y la investigación, pero tenían la dificultad de que no nos relacionaba con los temas y paradigmas que en nuestra ciencia se debatían en la comunidad sociológica internacional.

Con la apertura de la especialización a mediado de la década de los ochenta se mantienen algunos de los temas de los programas anteriores, se continúas utilizando el material de Stollberg, pero ahora se incorporan otra literatura y enfoques. Para esta fecha, circulan por el país los libros: Pasión por Excelencia y en busca de la Excelencia. ${ }^{7}$ Lo que indica la búsqueda de otros puntos de vista al analizar otras experiencias en el ámbito empresarial y/o docente. Se añaden autores nacionales y resultados de investigaciones de esta esfera en el país.

A partir de la reapertura del Departamento de Sociología en 1984 y de reiniciarse el proceso de institucionalización de esta ciencia en el país se hacen distintos intentos para establecer vínculos con otras instituciones que puedan contribuir al desarrollo de la carrera y la superación de su claustro de profesores. Esta actividad ha venido fructificando en la medida en que se consolida y se conoce las existencia de la formación de 4 sociólogos en el país. En 1994, fecha en que ya se imparte por segunda vez el programa de Sociologías del Trabajo en el nuevo Plan de Estudio, se realizan dos seminarios de posgrado en el Departamento de Sociología que han tenido mucha importancia para la vida académica de nuestra institución y otras

\footnotetext{
Stollberg, Aspectos sociológicos del trabajo, UH, 1986. Estos dos últimos libros, aunque publicados en la década de los ochenta fueron utilizados en el período que analizo. También se manejaron artículos de las revistas de Ciencias Sociales de la Academia de Ciencias de la URSS y Socialismo, Teoría y Práctica. En la literatura también se utilizó El capital de Kart Marx y trabajos de F. Engels y V. I. Lenin.

7 Estos libros se difundieron entre los cuadros del Sistema de Capacitación del Ministerio de la Industria Básica (MINBAS).
} 
áreas docentes y/o de investigación. El primero " Teoría y práctica de la metodología cualitativa aplicada a los procesos sociales y formas de identidad" ${ }^{8}$ y el segundo "Reorganización productiva y organización del trabajo en los países industriales centrales" desarrollada por el Dr. Juan José Castillo(septiembre de 1994), catedrático de la Universidad Complutense de Madrid. En el también participó Carlos Alá Santiago de la Universidad de Puerto Rico.

El seminario permitió que tuviéramos una visión acerca de las relaciones laborales en la década de los noventa. El papel del trabajo en los sistemas complejos; las grandes empresas y el proceso de "pequeñización interna"; el papel de la pequeña y mediana empresa; distritos industriales, redes de empresa y sistemas productivos locales; la innovación tecnológica y sus consecuencias sociales; así como sus reflexiones acerca de paradigmas, métodos y enfoques en Europa y América Latina a través de la Sociología del Trabajo. Las temáticas estuvieron avaladas por resultados de investigación y la metodología aplicada

Este curso nos dio un nivel de actualización importante y sentó las bases para continuar conociendo y aplicando los contenidos recibidos. La bibliografía utilizada en el posgrado fue donada al Departamento, lo que ha contribuido a enriquecer la docencia y la investigación en la Universidad y en las instituciones que participaron en el curso.

Como resultado del seminario, a finales de septiembre de ese año se constituyó el Taller de Sociología del trabajo que tiene como finalidad contribuir a fortalecer la cooperación, conocimiento y superación de los

\footnotetext{
8 Este posgrado se realizó en el Departamento de Sociología (UH) entre el 25 de abril al 11 de mayo de 1994, dirigido por los profesores José Arribas Machado (Universidad Nacional de Educación a Distancia, Madrid) y Cristina Santamarina (Universidad Complutense de Madrid). El seminario fue muy útil, pues permitió interiorizar una concepción más amplia y menos esquemática del proceso de investigación que la utilizada hasta ese momento por la mayoría de los investigadores y docentes. La incorporación bastante generalizada de la historia de vida y las entrevistas en profundidad. Las que se habían utilizado por los sociólogos, antropólogos y otros investigadores experimentados pero de forma aislada. Ahora estos métodos en muchos casos constituyen la única fuente de información o sirven para complementar la investigación.
} 
profesionales con la docencia y/o la investigación u otras actividades vinculadas a la esfera laboral. También pretende desarrollar una red de cooperación.

El taller puede ser un marco apropiado para estrechar más las relaciones con la sociedad Latinoamericana de Sociología del Trabajo y propiciar las investigaciones y superación de sus integrantes, mediante la realización de seminarios y talleres donde podamos conocer los resultados y desarrollos alcanzados en otros países o regiones y al mismo tiempo mostrar los procesos que hoy ocurren en el país.

La docencia de la Sociología del Trabajo ha sido predominantemente en pregrado. En estos momentos se encuentra muy avanzado el proceso de institucionalización de una maestría en sociología en la UH. En la misma, un área especialización será la Sociología del Trabajo.

En la UH, además del departamento de Sociología, encuentran otras dependencias que se dedican o incluyen la docencia en la esfera laboral, como son: Centro de Estudios de Técnicas de Dirección (CETED), Facultad de Psicología; Centro de Estudios Demográficos; Departamento de Estudios sobre el Desarrollo y la Facultad Latinoamericana de Ciencias Sociales (DES-FLACSO).

La dependencia de más experiencia sistematizada en posgrado en el área laboral es el CETED. Fundada en 1988. Este centro ha desarrollado diferentes cursos, talleres, seminarios, maestrías y otras actividades de posgrado. La maestría en dirección de este centro fue la primera aprobada en ciencias sociales en el país.

\section{Las investigaciones laborales en la Universidad de La Habana}

Las investigaciones laborales en la $\mathrm{UH}^{9}$ se han venido realizando de acuerdo a las distintas etapas por las que ha atravesado el país. Los temas

\footnotetext{
9 Solamente me refiero a las investigaciones de la UH. En la revista Economía y Sociología del trabajo, núm. 23/24 del Ministerio del Trabajo y Seguridad Social de España parece un artículo de Carlos Alá y José Luís Martín que se refiere a los estudios del trabajo en Cuba y Puerto Rico.
} 
estudiados hasta la década de los noventa, en su expresión más general, no coincidían con las problemáticas que en esta esfera se investigaban en el mundo y particularmente en América Latina. O. al menos, no se incluyeron expresamente temas de moda en un momento determinado. Esto se debe, como se señaló anteriormente, a la intermitencia de la institucionalización de esta ciencia en el país y al nivel de información e intercambio existente.

La investigación sociológica tanto macro como micro debe corresponder con las necesidades o demandas sociales del país. Sin embargo, hay que tener en cuenta los temas, paradigmas y experiencias que ocupan la reflexión sociológica tanto en los países capitalistas desarrollados como subdesarrollados. Un lugar de referencia lo constituyen, sin lugar a dudas, América Latina y el Caribe.

Las transformaciones que se producen en la sociedad cubana en la actualidad y los procesos a que dan lugar en la esfera laboral, nos aproximan a los estudios que se realizan en nuestra disciplina. En aspectos tales como: el redimensionamiento empresarial, la reconversión tecnológica y sus efectos en el mercado de trabajo; el impacto de las nuevas tecnologías y sus consecuencias en la calificación y las condiciones de trabajo; la toma de decisiones y la participación de los trabajadores; los sindicatos y su papel en la coyuntura actual, etcétera.

Las investigaciones laborales en la UH tienen su antecedente más cercano en los estudios realizados por el doctor Raúl Gutiérrez Serrano acerca de la productividad y las condiciones de trabajo en nueve centrales azucareras del país, entre 1969 y 1970.

El tema de los cuadros de dirección ha ocupado un lugar importante en la sociología y otras ciencias sociales, ${ }^{10}$ en la UH y otras instituciones. Durante varios años en que inicia la docencia de la Sociología del trabajo en

\footnotetext{
10 Esto se debe a la importancia que se le concede a la actividad de los cuadros desde los primeros años de la Revolución. E artículo de Ernesto Che Guevara, «El cuadro columna vertebral de la Revolución» es un ejemplo. C $f$. El Che en la Revolución cubana, La Habana, Ministerio del Azúcar, 1966, t. 1 pp. 1-6.
} 
la UH, dedica sus estudios a esta temática. Fruto de este trabajo es el libro: Formación científica y dirección desde el subdesarrollo (Bermúdez, 1983). Donde se fundamenta el carácter especializado y creador de esta actividad.

La investigación y la docencia en esta temática se incrementa a partir de la realización del Primer Congreso del PCC, en 1975 y la aprobación de las resoluciones: «Sobre el sistema de dirección y planificación de la economía» y «Sobre la política de formación, selección, ubicación y promoción y superación de los cuadros». ${ }^{11}$

Al calor de esta política se crea el CETED de la UH en 1988, con los objetivos de crear cuadros y personas que trabajaran en la preparación de cuadros. Así se formaron más de 400 profesionales. Se calificó también a unos 2500 dirigentes anuales, de Empresas y organismos de la economía interna. ${ }^{12}$

Otra línea sistematizada en el área laboral la constituye el estudio desarrollado por el Centro de Estudios para el Perfeccionamiento de la Educción Superior de la UH (CEPES), acerca del proceso de distribución, formación, adaptación y utilización de los egresados de la educación superior. La investigación ha venido brindando importantes resultados acer4ca del proceso de reproducción de la fuerza de trabajo calificada en el país, principalmente en la esfera productiva por el valor estratégico que éstos tienen para el desarrollo presente perspectivo del país. ${ }^{13}$

11 C $f$. Tesis y resoluciones del Primer Congreso del Partido Comunista de Cuba, Dep. de Orientación Revolucionaria del Comité Central del Partido Comunista de Cuba, La Habana, 1976.

12 De la entrevista realizada al doctor Carlos Díaz Llorca, subdirector del CETED. En 1988 se crea, además, el Grupo de Aspectos Sociopsicológicos de la Dirección (PSIDIR), constituido por profesores de la Facultad de Psicología y por psicólogos pertenecientes a diferentes organismos de la esfera productiva y de servicios que desempeñan sus funciones profesionales vinculados a la actividad de dirección. Desde la perspectiva de la Psicología de la Dirección se realizan investigaciones, seminarios, talleres y cursos de posgrado con vistas a la optimización de la dirección empresarial y otras organizaciones sociales.

13 La investigación se realiza desde la década de los ochenta. Tiene un carácter multidisciplinario y es dirigida por el profesor Enrique Iñigo. En sus inicios tomamos parte algunos integrantes del Departamento de Sociología. El estudio constituyó un tema del Programa Científico-Técnico acerca de la formación de la juventud desarrollada en el quinquenio de 1986-1990. En la introducción de los resultados han interactuado el Ministerio de Educción Superior (MES); el Ministerio del Trabajo y Seguridad 
Otras investigaciones desarrolladas en la esfera laboral desde el Departamento de Sociología de la UH han estado encaminadas a dar solución, a pericón de instituciones acerca de la disciplina laboral, fluctuación (rotación), problemas institucionales o de mercado laboral.

En el quinquenio 1986-1990 en el Programa Científico-Técnico de la Juventud se le encargó al Departamento de Sociología el estudio de los adolescentes y jóvenes desvinculados del estudio y del trabajo. Con una muestra nacional se estudiaron un conjunto de factores concurrentes del mercado de trabajo, de la estructura social, institucionales y sociales. Los resultados obtenidos contribuyeron a la integración del informe final del Programa (Catá, González, Ibarra et all, 1990).

En 1986 se desarrolló el trabajo de investigación: «Estudio sociopolítico de los factores principales que inciden en las condiciones de trabajo en el Centro de Operaciones Luís Felipe Almeida»; ${ }^{14}$ perteneciente al Ministerio de Industria Básica. Aquí se identificaron algunos problemas propios del centro, en el orden de las condiciones de trabajo, aspectos organizativos y de dirección; contradicciones entre la unidad y la empresa; problemas de retención de personal en determinados puestos de trabajo como el de los linieros; dificultades a partir de la estructura del centro. Otros que se vinculaban a situaciones generales y que tenían que ver con el modelo económico imperante, como era el fondo de estimulación y su utilización; formalidad en los organismos colectivos de participación establecidos. Como elementos positivos, la existencia de un fuerte movimiento de innovadores y racionalizadores que aseguraban el funcionamiento de la producción del centro.

Entre los años 1990 y 1992 se realizaron diferentes acciones en la Empresa de Uniones Soldadas, Cubana de Acero, perteneciente al Ministerio

Social (MINTRAB); los Organismos de la Dirección Central del Estado (OACE), y la Academia de Ciencias, actual Ministerio de Tecnología y Medio Ambiente.

14 C $f$.Euclides Catá, Clotilde Proveyer, Reina Fleitas et al., Informe de Investigación. 
de la Industria Sideromecánica. El estudio tuvo carácter institucional y tomaron parte en él, además del equipo del departamento, estudiantes de diferentes años. Tres hicieron allí sus trabajos de tesis de diploma para la especialización en sociología. Se le entregaron al centro: 1) Un diagnóstico de las causas que influían en la fluctuación laboral de la empresa (Cata, Fleitas, Urrutia et al, 1991), 2) Caracterización de la fuerza de trabajo de los talleres de la Empresa y 3) Se elaboró conjuntamente con la empresa u «Plan de orientación profesional para los jóvenes de nuevo ingreso al centro» Los resultados tuvieron aplicación parcial, pues del estudio quedó su significado metodológico. En fecha inmediata los efectos del período especial provocaron cambios sustanciales en el proceso productivo del centro y en la fuerza de trabajo.

En la segunda mitad de 1994, se realiza un estudio de caso en la industria cigarrera con vistas a analizar las causas del absentismo laboral, y conocer desde sus inicios los efectos que iban teniendo las medidas que se estaban aplicando en el país en la conducta y desarrollo de las relaciones laborales en ese centro. A este trabajo se le ha venido dando seguimiento (Catá, 1994).

Desde mediados de 1995 realizamos una investigación comparada entre instituciones docentes de Cuba, España, México y Argentina acerca de las políticas económicas y su efecto en las relaciones laborales.

Otras instituciones, algunas ya mencionadas, realizan estudios laborales. EI CETED ha dirigido sus investigaciones hacia la "tropicalización" de las técnicas de dirección y su aplicación a las distintas empresas del país; tales como: marketing, calidad total, liderazgo y comunicación, solución de conflictos, etcétera.

El Centro de Estudios Demográficos (CEDEM) ha realizado estudios con el fin de aportar elementos para la formulación de políticas y estrategias de desarrollo en las que se incluyen las características de la población como recurso laboral y otras más específicas sobre: fluctuación laboral, trabajo por cuenta propia y en la caracterización de las Unidades Básicas de Producción Cooperativa (UBPC). 
En la Facultad de Psicología hay un grupo de estudios de aspectos sociopsicológicos de la dirección, que ha realizado varias investigaciones relacionadas con esta temática. El Centro de Estudio de la Economía Cubana $(\mathrm{CEEC})^{15}$ realiza investigaciones de la economía cubana y consultoría de empresas.

En las distintas experiencias de la enseñanza de la sociología en el país, se ha incluido la práctica laboral, la investigación y la docencia. Lo que se ha aplicado de acuerdo al diseño del plan de estudio, las condiciones económicas del país, las temáticas de investigación de la institución y la demanda social. ${ }^{16}$ En el actual plan de estudios se relaciona la práctica laboral y la investigación a través de los talleres sociológicos.

De acuerdo con el recuento realizado podemos ver que en las investigaciones laborales en la UH ha predominado el análisis micro dirigido a esferas parciales de la sociedad. No ha existido en esta área reflexiones sistematizadas acerca del devenir de las relaciones laborales en el país y los factores o causas que los expliquen. Las tendencias que se observan en la institucionalización de esta ciencia en la sociedad cubana — reapertura de la carrera, estudios de posgrado, publicaciones, realización y participación en eventos nacionales e internacionales y otras acciones que permitan la creación de la Asociación Nacional de Sociología; además de mantener y

\footnotetext{
15 El CEE se funda en 1989. Desde entonces han funcionado dos grupos. Uno que se dedica al estudio de la economía en Cuba y otro dedicado a la consultoría general. Ese último ha venido sistematizando resultados y experiencias que se están plasmando en tesis de maestrías y la conformación de una Maestría en Desarrollo Organizacional y Consultoría.

16 Se relacionan los trabajos de diploma asociados a la temática laboral de los graduados como especialización de sociología a partir de la carrera de filosofía y de la licenciatura actual en sociología. María Hernández León, «Caracterización del desarrollo de la adaptación en los egresados en la Educación Superior. 12-7-1989»; Gladis M. Núñez D., «Estudio de la fluctuación laboral potencial de los jóvenes vinculados a la producción y de servicios en la Empresa Constructora de Ómnibus Claudio Argüelles y Astillero Chullima», 12-2-1989; Maité Montalvo Mendoza, «El proceso de recalificación y el ejercicio de la plena igualdad de la mujen», 3-7-1990; Marta Oneida Pérez C., «Estudio sobre el desarrollo laboral de los graduados de Ciencias Técnicas y Agropecuario», 4-7-1990; Nelys García Blanco, «Caracterización de la adaptación a la vida laboral de jóvenes trabajadores en la Empresa Cubana de Acero», 1991; Tania Garmendía C., «La participación social de los trabajadores en la esfera laboral en la Empresa Cubana de Acero», 2-5-1991; Carlos Manuel Rodríguez A.; «Investigación sobre la fluctuación potencial de la fuerza de trabajo joven en Cubana de Acero», 1991, y Orlando Ruz, «La eficiencia de las asambleas por la eficiencia económica», 2212-1995.
} 
sistematizar estudios de casos o esferas parciales, conllevará a la realización de trabajos más abarcadores.

\section{Perspectivas de la Sociología del Trabajo en la Universidad de La Habana}

El rejuvenecimiento de la Sociología del Trabajo ${ }^{17}$ en América Latina se evidencia en la actividad de su comunidad científica para interpretar y explicar las transformaciones que se dan en la región como consecuencia tanto de políticas nacionales como la influencia que tienen en el mundo del trabajo procesos macro como la globalización de la producción.

En Cuba en la actualidad se producen transformaciones en las relaciones laborales, que tienen similitud con los procesos que ocurren en los países industriales centrales y especialmente en América Latina, lo que encuentra su expresión en el establecimiento de nuevas formas de organización del trabajo y de dirección de la producción, los cambios tecnológicos y productivos y su efecto en la fuerza de trabajo. El papel de los distintos actores sociales y la actuación de sus representantes.

Aunque es cierto que ocurren fenómenos similares entre nuestros países, es necesario contextualizar los procesos que hoy suceden en la sociedad cubana; sus particularidades y manifestaciones en la esfera local. En este empeño hay que conjugar las acciones de otras ciencias sociales tanto desde el análisis de sus propios objetos de estudio como en investigaciones multidisciplinarias. A la sociología le corresponde la explicación de lo típicamente social y la función integradora.

Uno de los aspectos a tener en cuenta es el contenido del nuevo modelo económico que se ha venido formando y su influencia en las relacio-

17 C $f$. Jorge Carrillo C., «La Sociología del Trabajo. En América Latina y su primer Congreso», Sociología del Trabajo, núm.20. 
nes laborales. La forma en que se manifiesta la relación centralizacióndescentralización, la democratización en la dirección, el sentimiento de pertenencia de los trabajadores, y la estimulación material y moral.

El tema de los sindicatos y su papel en la sociedad, poco estudiado hasta ahora, adquiere relevancia ante la necesidad de que tome un nuevo protagonismo ante los cambios que ocurren en la esfera laboral. El problema de la inversión extranjera, la legislación laboral y el derecho de los trabajadores son de vital importancia en estos momentos, así como su participación en la elaboración y control de convenios colectivos de trabajo, tanto en la empresa de distintas modalidades de capital extranjero como estatales.

El estudio de la inserción en la economía cubana de la microempresa, pequeña y mediana empresa. Tema que hasta la reforma de los noventa no constituía un problema de reflexión sociológica por ser prácticamente inexistente.

Las características del proceso de ajuste en Cuba y la necesaria, pero al mismo tiempo contradictoria, vinculación entre eficiencia productiva y las políticas sociales.

Mercado de trabajo y racionalización junto a lo anterior son los temas actuales de la Sociología del Trabajo.

Aquí sólo menciono algunos temas a estudiar. Su identificación, unida a otros temas no relacionados, además del recuento realizado nos indica los retos que tiene la Sociología del Trabajo en la UH y el país.

Esto nos señala el esfuerzo a realizar en cuanto a la necesidad de desarrollar la metodología de la investigación, las técnicas y métodos de recogida de la información, y ampliar el horizonte reflexivo acerca de la complejidad que adquiere la sociedad cubana y su efecto en las relaciones laborales.

En este sentido, recibimos la idea de que: «El futuro de la Sociología del Trabajo está en su fuerza analítica, conceptual, de diseño epistemológico, 
capaz de hacer aparecer bajo las capaz de apariencia cada vez más densas, duras y espesas, las relaciones sociales que están en su base»(CASTILLO, 1996, p. 419).

\section{Referências}

BERMÚDEZ, Ricardo Machado. Formación de cuadros y dirección científica desde el subdesarrollo. La Habana: Ciencias Sociales, 1983.

CASTILLO, Juán José. El trabajo del sociólogo. Madrid: Complutense, 1996.

CATÁ, Euclides. Estudio de los factores que inciden en el ausentismo laboral en la fábrica de Cigarros Populares. Informe de Investigación, Dep. de Sociología, diciembre de 1994.

CATÁ, Euclides; FLEITAS, Reina; URRUTIA, Lourdes et al.. Estudio preliminar en la Empresa Cubana de Acero sobre las causas que influyen en la fluctuación potencial. Informe de Investigación, Departamento de Sociología, diciembre de 1991.

CATÁ, Euclides; GONZÁLEZ, Ernel; IBARRA, Francisco et al., Perfeccionamiento del trabajo con adolescentes y jóvenes desvinculados del estudio y del trabajo. Informe de Investigación, Departamento de Sociología, 1990.

CATAÑO, Gonzalo. La Sociología en Colombia. Plaza y Janés, 1986.

CATAÑO, Gonzalo. Sociología del Trabajo. nueva época, núm. 29, invierno de 1996/1997. p. 161-173.

CECILIOA BOBES, Velia. La Sociología en América Latina. Notas para una periodización. La Habana: Ciencias Sociales, 1990. 


\section{Resumo}

El artículo da una breve panorámica del desarrollo de la Sociología en Cuba. De forma particular se realiza un análisis de la docencia de la Sociología del Trabajo en la Universidad de la Habana, enfoques, áreas de influencia y las instituciones que han participado en la docencia e investigación de la esfera laboral en Cuba. Finalmente se dedica un espacio a las perspectivas y retos de la Sociología del Trabajo en Cuba.

Palabras-clave: Sociología en Cuba, sociología del trabajo, investigaciones laborales, temas sociología del trabajo. 


\section{Sociology of labor at the University of Havana}

\section{Euclides Catá Guilarte}

The article is a brief overview of the development of Sociology in Cuba. In a particular way, an analysis is conducted about Sociology of Labor in the University of Havana, its approaches, areas of influence, as well as the institutions that took part in teaching and research within the labor field in Cuba. Finally, the perspective and directions of Sociology of Labor in Cuba are examined.

Key words: sociology in Cuba, sociology of labor, labor research, themes in sociology of Labor. 